\title{
Stem cells in urology: how far have we come?
}

\author{
Ching-Shwun Lin and Tom F Lue
}

Stem cell research in urology can be broadly subdivided into two fields: one that attempts to identify urological tissue-specific stem cells; and another that tries to apply lessdifferentiated multipotent stem cells, such as embryonic stem cells (ESCs), to the treatment of urological diseases. Several studies have investigated tissue-specific stem cells in the kidney, bladder, prostate, penis and testes. The main obstacles in this field of research are the lack of reliable markers for putative tissue-specific stem cells, and the absence of a convincing rationale to justify their use over less-differentiated stem cells, which offer more therapeutic flexibility and are usually more-easily accessible.

In addition to tissue-specific stem cells, ESCs and some 'adult' multipotent stem cells have been investigated for urological applications. These adult types include bone marrow stem cells (BMSCs), skeletal-muscle-derived stem cells (SkMSCs), adipose-tissue-derived stem cells (ADSCs), and amniotic-fluid-derived stem cells (AFSCs). Although ESCs and BMSCs are the best studied stem cells in most medical disciplines, SkMSCs are the only type to have reached clinical trials in urology. These trials were conducted by Michael Chancellor's group at the University of Pittsburgh in the US and Hannes Strasser's group at the Medical University of Innsbruck, Austria. However, as Nature has been reporting (Abbott A [2008] Nature 454: 922-933), questions have been raised about the ethics and methodology of the Austrian studies. At the time of writing full details of the issues surrounding these studies have yet to be publicly disclosed.

A wide range of studies have focused on urological applications of multipotent stem cells. ESCs, BMSCs and AFSCs have been shown to differentiate into renal lineages and to enhance renal repair. With regard to the ureter, tissue engineering has been attempted but

\section{Despite these \\ advances, challenges \\ abound. \\ Many such \\ difficulties, \\ however, are \\ not unique \\ to urology}

C-S Lin is Director

of the Molecular

Urology Laboratory

and TF Lue is

Professor and Vice

Chair of Urology,

at the University

of California, San

Francisco, CA, USA.

\section{Competing interests}

TF Lue has declared

an association with the

following organization:

National Institutes of Health.

See the article online for full

details of the relationship.

C-S Lin declared no

competing interests.

www.nature.com/clinicalpractice doi:10.1038/ncpuro1219 studies on stem cells are still lacking. BMSCs, SkMSCs, and AFSCs have been used for bladder augmentation and detrusor regeneration in animals. SkMSCs are the only stem cells to have been successfully tested in humans, for the treatment of stress urinary incontinence. ESCs, BMSCs and SkMSCs have been shown to improve erectile function in animal models. Both ESCs and BMSCs can be differentiated into sperm and, remarkably, the ESC-derived sperms have generated offspring mice. With regards to the prostate, stem cell studies are usually focused less on the application of stem cells to treat disease, and more on understanding so-called 'cancer stem cells', which are targets of therapy.

ADSC research is a relatively young field, and these cells are largely unstudied in urology. However, as a result of their high differentiation potential and ease of isolation, ADSCs represent an exciting resource for tissue engineering and regenerative medicine within and beyond urology.

Despite these advances, challenges abound. Many such difficulties, however, are not unique to urology. Concerns have been raised about the ethical issues surrounding the use of ESCs, and their potential tumorigenicity. Unanswered questions also remain. Can adult stem cells really transdifferentiate and thus replenish degenerated tissue? Or do they simply secrete growth factors that help the host tissue to regenerate? More importantly, how translatable are the results of preclinical studies in largely healthy animal models to clinical situations in which human patients often have considerable comorbidity? Notably, these questions might best be answered by research in urology, rather than in other disciplines, because most urological organs are structurally simple and easily accessible. If we recognize the potential of this technology, urological stem cell research should have a bright future. 